\title{
Das Gipsmodell als eigenständiges Kunstwerk
}

\author{
Oberlandesgericht Rostock, Urteil vom 05. März 2007 - 3 U 103/06
}

An einem im Rahmen der Erarbeitung eines Kunstwerkes gefertigten Gipsmodell, in welches eine eigene schöpferische Tätigkeit des Urhebers eingeflossen ist, bestehen ein von der Übereignung des fertigen Kunstwerkes unabhängiges eigenständiges Eigentumsrecht sowie eigenständige Urheber- und damit Verwertungsrechte. Wird ein solches Modell ausgestellt, liegt dem regelmäßig ein Leihvertrag zu Grunde. (Leitsätze d. Redaktion)

$[\ldots]$

\section{Gründe:}

\section{I.}

- Der Kläger begehrt von dem beklagten Land Schadensersatz wegen der Zerstörung eines von ihm gefertigten Gipsmodells. Es handelt sich dabei um ein lebensgroßes Modell der Bronzestatue eines Knaben auf einem Pferd. Der Kläger hatte sie im Auftrag der Bundesrepublik Deutschland erstellt, die im Rahmen der Organleihe durch die Oberfinanzdirektion R. (nachfolgend OFD) vertreten wurde. Das Gipsmodell wurde 1996 im Lichthof des Hauses 3 der ehemaligen Oberfinanzdirektion R. aufgestellt. Im November 2002 wurde es beschädigt, wobei die Umstände, die zu den Beschädigungen führten, zwischen den Parteien streitig sind. Zerstört oder beschädigt wurden drei Beine und der Schweif des Pferdes sowie Kopf, Arme und Füße des Reiters. Das Modell, so behauptete der Kläger, sei im Laufe von Umbauarbeiten in der OFD beschädigt worden, da diese keine geeigneten Schutzmaßnahmen für das Modell ergriffen habe.

Das beklagte Land wandte ein, die Aufstellung des Gipsmodells in der OFD sei nur aus Gefälligkeit erfolgt, weil der Kläger nicht gewusst habe, wo er es unterbringen sollte. Bei den Gesprächen hierzu habe der Zeuge T. den Kläger darauf hingewiesen, dass eine versicherungstechnische Absicherung nicht bestehe und eine Haftung nicht übernommen werde.

Das beklagte Land berief sich darauf, nicht passiv legitimiert zu sein, da die OFD auch bei der Zustimmung der Aufstellung des Modells im Lichthof des Hauses 3 der OFD nur in Organleihe für die Bundesrepublik Deutschland gehandelt habe. Auch sei nicht der Kläger, sondern die BRD als Auftraggeberin des Bronzegusses Eigentümer des Gipsmodells gewesen. Diese habe entsprechend der Wettbewerbsbedingungen auch das Eigentum an dem Gipsmodell erworben. Dem Kläger sei zudem kein Schaden entstanden, denn der Herstellung weiterer Abgüsse von dem Modell hätte die BRD nicht zugestimmt.

Mit Urteil vom 09.05.2006 - 100 352/05 - wies das Landgericht Rostock die Klage ab. Zur Begründung führte es aus, dass es dahinstehen könne, ob es sich vorliegend um ein Leih- oder ein Gefälligkeitsverhältnis handele, denn in beiden Fällen sei die Forderung des Klägers verjährt.

Mit seiner Berufung greift der Kläger das erstinstanzliche Urteil in vollem Umfang an. Das Landgericht habe zu Unrecht Verjährung angenommen. Das Werk sei nicht nur beschädigt, sondern zerstört, weil es nicht wiederhergestellt werden könne. Die Zerstörung des Kunstwerkes liege darin, dass der gesamtkünstlerische Charakter und damit der individuell künstlerische Ausdruck verloren gegangen sei. Ein Abdruck etwa der fehlenden Teile von der Bronzeskulptur in P. könnte nicht zur Reparatur des Modells führen, denn bei der Abformung in Bronze schrumpfe die Skulptur um $3 \%$ bis $4 \%$. Daher wären Teile, die von der Bronzeskulptur abgeformt werden, zu kurz. Es könnten zwar neue Teile, etwa Beine, modelliert werden. Dadurch würde aber nicht das alte Kunstwerk wiederhergestellt, sondern ein neues Kunstwerk geschaffen. Daher greife bereits die Verjährungsfrist des § 606 BGB nicht.

Schuldhaft habe das Land es unterlassen, die Skulptur vor herabbrechenden Bauteilen, die diese beschädigt haben, zu schützen, wobei das Verschulden gem. § 280 Abs. 1 BGB vermutet werde. Wenn das beklagte Land behaupte, das Pferd sei unbeschadet am 13.11.2002 in das Haus 2 verbracht und dort in der Nacht von Unbekannten beschädigt worden, liege hierin die Verletzung einer Verkehrssicherungspflicht, die ebenfalls eine Schadensersatzpflicht begründe.

Den Anspruch könne der Kläger zudem auf die Verletzung des urheberrechtlich geschützten Werkes (§ 97 Abs. 1 Satz 1 Urheberrechtsgesetz) stützen. Der Kläger sei Urheber. Die Skulptur stehe als Werk i.S.d. § 2 Abs. 1 Nr. 4 UrhG unter dem Schutz des UrhG. Dem stehe der Umstand, dass es sich um eine Gipsskulptur handele, von der bestimmungsgemäß Bronzen gefertigt werden sollen, nicht entgegen. Geschützt seien auch Entwicklungsstadien von Werken und bloße Entwürfe. Es komme nur darauf an, ob sich in dem Werk eine persönlich geistige Schöpfung mit einem Mindestmaß an Schöpfungshöhe findet. Unter den Schutz des § 97 UrhG falle - so § 15 UrhG - auch das Recht des Künstlers, das Werk wirtschaftlich zu verwerten, so etwa für weitere Abgüsse. Der Beklagten sei zumindest die fahrlässige Verletzung des Werkes vorzuwerfen.

Die Höhe des Schadens ergebe sich daraus, dass der Kläger nunmehr konkrete Angebote erhalten habe, das Gipsmodell anderweitig auszustellen. Für diese Ausstellungen wäre es mindestens in Höhe des hier geltend gemachten Schadens versichert worden. Gipsmodelle anderer Künstler würden in renommierten Ausstellungshäusern ausgestellt. Der Kläger hätte von diesem Modell aber auch weitere Abgüsse fertigen können, die ihm einen Gewinn in mindestens der geltend gemachten Höhe erbracht hätten.

Ansprüche des Klägers auf Ersatz des immateriellen Schadens nach § 97 Abs. 2 Urh G habe das Gericht nicht geprüft. Mit der Zerstörung des Werkes habe die Beklagte gleichzeitig Urheberpersönlich- 
keitsrechte verletzt. Dem Kläger wäre zumindest ein immaterieller Schadensersatz in Höhe von 6.000,00 € zuzuerkennen. Bei der Bestimmung der Schadenshöhe sei das Verschulden des Verletzers, die persönliche Bindung des Künstlers zu dem Werk, dessen Bekanntheitsgrad, Art und Umstände der Verletzung zu berücksichtigen. Der Kläger sei über die Grenzen des Landes M.-V. anerkannt. Die Anfertigung eines solchen Gipsmodells dieser Größe benötige in der Regel ein Jahr. Es stelle eines der herausragendsten Werke des Klägers dar, zu dem eine enge Bindung bestehe. Vergleichbare Werke dieser Größe gebe es vom Kläger nur noch drei. Der Knabe auf dem Pferd habe zudem seinen 13-jährigen Sohn dargestellt.

Das beklagte Land hält die Verjährungseinrede aufrecht und ist der Ansicht, dass die Verjährung bei der Leihe und dem Gefälligkeitsverhältnis bereits mit dem Zeitpunkt zu laufen beginne, zu dem der Verleiher die Möglichkeit hat, die vollständige Sachherrschaft über die Sache wiederzuerlangen, nicht erst bei Rückgabe. Es behauptet, sämtliche Teile der Skulptur seien noch vorhanden.

Die Skulptur habe unter einer vorspringenden Decke gestanden, sei mit Planen eingehaust worden und habe daher auch keine Beschädigungen von oben erfahren können. Bei Abbruch des alten Fußbodenbelages sei auf die Skulptur besonders geachtet worden. Um die Arbeiten abschließen zu können, sei es erforderlich gewesen, die Skulptur kurzzeitig aus dem Lichthof zu verbringen. Am 13.11.2002 hätten sechs Mitarbeiter die noch unbeschädigte Skulptur vom Haus 3 in das Haus 2 gebracht. Die Handwerker hätten die Skulptur unbeschädigt in Haus 2 gebracht und abgestellt. Es habe im Haus 2 am 13.11.02 abends und am 14.11.02 keine Bautätigkeiten gegeben. Am 14.11.02 morgens seien die Beschädigungen der Skulptur festgestellt worden. Es fehle, da die Mitarbeiter der OFD die im Verkehr erforderliche Sorgfalt hätten walten lassen, an Vorsatz oder Fahrlässigkeit. Zudem sei zwischen dem Kläger und dem Finanzpräsidenten a.D. T. ein Haftungsausschluss vereinbart worden.

Das beschädigte Modell könne wieder hergestellt werden. Der Kläger habe es in Einzelteilen ausgeliefert und dann zusammengesetzt. Der Kläger habe keinen Verkaufserlös aus weiteren Abgüssen erzielen können, so dass ihm ein Schaden nicht entstanden sein kann, weil die BRD eine erforderliche Zustimmung hierzu nicht erteilt hätte. Auch im Weiteren bestreitet das Land den Wert der Gipsskulptur, die Möglichkeit, diese nunmehr auszustellen sowie die Höhe eines möglichen Erlöses aus der Veräußerung weiterer Abgüsse.

Das beklagte Land meint, Ansprüche des Klägers scheiterten an seiner Sorgfaltspflichtverletzung. Zunächst hätte er beim Aufstellen der Skulptur auf die Verletzlichkeit des Werkstoffes hinweisen müssen. Zudem habe der Zeuge O. dem Kläger mitgeteilt, dass in der OFD demnächst Bauarbeiten stattfinden würden und er sich doch um sein Pferd kümmern solle. (Sachverhalt von der Redaktion gekürzt.)

\section{II.}

Die zulässige Berufung des Klägers hat Erfolg. Der Senat sieht einen Schadensersatzanspruch dem Grunde nach als gegeben, dessen Höhe jedoch noch festzustellen ist.
1. Der Kläger hat einen Schadensersatzanspruch gem. $§ \S 598 \mathrm{ff.}$, $\S 280$ BGB wegen Verletzung von Obhuts- und Verkehrssicherungspflichten aus einem Leihvertrag.

a) Wird ein Kunstobjekt gezielt zum Zwecke der Ausstellung an einen Aussteller übergeben, handelt es sich regelmäßig um einen Leihvertrag (Loewenheim, Handbuch des Urheberrechts, 2003, § 70 Rn. 28; OLG Düsseldorf, Urt. vom 02.08.1988 - 7 U 268/87 - NJW 1990, 2000; OLG Naumburg, Beschl. vom 29.12.2005 - 2 W 14/05 OLGR 2006, 969). Das ist der Fall, wenn dem Aussteller von vornherein das Ausstellungsrecht zugebilligt wird, ihm also unentgeltlich der Gebrauch gewährt wird. Übernimmt dagegen der Aussteller nur die Ausstellung eines wirtschaftlich unbedeutenden Gegenstandes, kann darin eine Gefälligkeitshandlung liegen (OLG Brandenburg, Urt. vom 23.09.2003 - 2 U 9/03 - NJW 2004, 620 für eine aus einem Bierfass von einem Schüler im Unterricht gefertigte Bar). Steht dagegen die Aufbewahrung des Kunstobjektes im Vordergrund, handelt es sich selbst dann um einen Verwahrvertrag, wenn demjenigen, dem das Objekt überlassen wird, jeweils nach Rücksprache mit dem Überlasser das Recht eingeräumt werden soll, das Kunstobjekt gelegentlich auch auszustellen (OLG Naumburg, a.a.O.). Welches dieser Rechtsverhältnisse im Einzelfall vorliegt, ist nach den Umständen des Einzelfalles und dem wesentlichen Zweck des Vertrages zu beurteilen (OLG Brandenburg, a.a.O.).

Im Ergebnis der Würdigung der Umstände des Falles nimmt der Senat ein Leihverhältnis an. Die Skulptur wurde für jedermann deutlich sichtbar im Lichthof des Hauses 3 der OFD aufgestellt und damit Mitarbeitern als auch Besuchern des Hauses die Möglichkeit eröffnet, sie anzusehen. Um sie etwaigen Besuchern zeigen zu dürfen, bedurfte die OFD keiner jeweiligen Zustimmung des Klägers, somit scheidet nach den oben skizzierten Grundsätzen ein Verwahrverhältnis, ohne dass es auf die Motivation beider Seiten zum Vertragsschluss ankommt, bereits aus. Ein Gefälligkeitsverhältnis scheitert bereits an dem Umstand, dass es sich bei der Gipsskulptur nicht um ein unbedeutendes Werk handelte. Zum einen genoss der Kläger bei Übergabe des Modells und erst recht bei Beschädigung des Modells einen beachtlichen Ruf als Künstler. Zum anderen verkörpert das Modell erhebliche künstlerische Tätigkeit, für deren Ergebnis in Form eines Bronzeabgusses die Bundesrepublik, die diese an repräsentativer Stelle aufstellte, ein nicht unbeachtliches Honorar entrichtete.

b) Auf die Eigentümerstellung des Klägers an dem Gipsmodell, die das beklagte Land bestreitet, kommt es für den klägerischen Anspruch nicht an. Der Leih- oder Gefälligkeitsvertrag setzt die Eigentümerstellung dessen, der die Sache verleiht oder anderweitig auf vertraglicher Grundlage einstellt, nicht voraus. Vielmehr kann er hierzu auch aus einem Nutzungs- oder sonstigen Besitzverhältnis befugt und in der Lage sein. Zudem hat die Bundesrepublik Deutschland auch nur das Eigentum an der beauftragten Bronzeskulptur erlangt, nicht auch an den zur Vorarbeit erforderlichen Materialien und Modellen. Ein im Rahmen der Erarbeitung eines Kunstwerkes gefertigtes Modell, in welches eine eigene schöpferische Tätigkeit des Künstlers/Urhebers eingeflossen ist, stellt ein von der Übereignung nicht erfasstes eigenständiges Eigentum, an welchem sogar eigenständige Urheber- und damit Verwertungsrechte bestehen 
(für plastische Darstellungen und Modelle Möhring/Nicolini, Urheberrechtsgesetz, 2. Aufl., § 2 Rn. 37; für die digitalisierte Grundsubstanz der Fertigung von Kartenmaterial BGH, Urt. vom 23.06.05 - 1 ZR 227/02 - BGH-Report 20051546 [LS] = GRUR 2005, 854) dar. Dem stünde es entgegen, wenn mit dem Vertrag zwischen Künstler und Auftraggeber auch sämtliche Modelle und Entwürfe übereignet worden wären. Hierfür gibt der Vertrag jedoch nichts her.

c) Im Ergebnis der Beweisaufnahme vom 29.01.2007 steht es für den Senat auch fest, dass die unstreitigen Beschädigungen ursächlich auf eine Verletzung der aus dem Leihvertrag resultierenden Sorgfalts- und Obhutspflichten zurückzuführen sind. Wer ein Kunstwerk entleiht, übernimmt damit die Verpflichtung, es bei Auf- und Ausstellung und Repräsentation vor Beschädigungen von Außen und durch Dritte zumindest insoweit zu schützen, wie dies mit dem Zweck der Präsentation gegenüber der Öffentlichkeit oder zumindest eines bestimmten Personenkreises vereinbar ist.

Die Skulptur wurde am 13.11.2002 vom Lichthof des Hauses 3 in das Haus 2 verbracht. Ebenso kann der Senat davon ausgehen, dass das Gipsmodell zu diesem Zeitpunkt noch unbeschädigt war. Das haben die Zeugen, soweit sie überhaupt über eigeneWahrnehmungen verfügten, bezeugen können. Die Angaben der Zeugen P. und T. sprechen zudem dafür, dass die Beschädigungen des Modells in der Nacht vom 13. zum 14.11.2002 durch unbekannte Dritte erfolgten. Die Angaben des Zeugen O. stehen dem nicht entgegen. Dass das Modell gänzlich unbeschädigt in das Haus 3 zurückgekehrt wäre, hat auch dieser nicht ausgesagt. Soweit er angab, die Beschädigungen hätten in der Folgezeit zugenommen, mag dieses zum einen sein subjektiver Eindruck gewesen sein. Zum anderen verwies er in seiner Vernehmung wiederholt darauf, dass es sich bei seinen Angaben um das handele, woran er sich noch erinnern könne. Insoweit er selbst einräumte, sich an Details überwiegend nicht erinnern zu können, sieht der Senat zu den Aussagen der vernommenen Zeugen keinen Widerspruch. Allerdings haben die Zeugen O., P. und T. sämtlich eingeräumt, dass das Haus 2 zu diesem Zeitpunkt eine weitgehend ungesicherte Baustelle war. Der Zeuge P. beschrieb den Zustand des Hauses 2 so, dass sämtliche Fenster und Türen herausgerissen und die Öffnungen nicht verschlossen worden waren. Das Haus war von außen eingerüstet und nur das Gerüst mit einer Plane abgehängt, so dass jederzeit Unbefugte das Gebäude unter der Plane hindurch und über das Gerüst durch die unverschlossenen Maueröffnungen betreten konnten. Dies deckt sich mit der Aussage des Zeugen O., dass im Haus 2 alles offen gewesen sei. Auch die Angaben des Zeugen T., dass die Fenster herausgerissen und mit Folien abgedeckt waren, stimmt damit im Wesentlichen überein.

Mit der Verbringung eines werthaltigen und zerstörungs- bzw. beschädigungsanfälligen Kunstwerkes in einen ungesicherten Baustellenbereich, der unbewacht und unkontrolliert des nachts für Dritte betretbar ist, hat das beklagte Land seine Obhuts- und Sicherungspflichten verletzt. Nach Auffassung des Senates hätte es dem beklagten Land zumindest oblegen, das Kunstwerk so zu sichern, dass es vor Übergriffen und Beschädigungen durch unbefugte Dritte hinreichend geschützt ist, indem etwa nur ein kontrollierter Zugang zur Ausstellungsfläche besteht. Ob aber die Beschädigung des
Modells durch Unbefugte erfolgte oder ob sich diese - wie dies der Zeuge O. angab - über mehre Tage hinzog, braucht der Senat letztlich nicht aufklären, weil auch dann das beklagte Land seiner Sicherungspflicht nicht genügt hätte. Es hätte ihm in diesem Fall oblegen, zumindest für einen so hinreichenden Schutz zu sorgen, dass das Kunstwerk während der Bauarbeiten keinerlei Schaden nimmt. Dass es dem nicht genügt haben kann, drängt sich dem Senat unter Zugrundelegung der Angaben des Zeugen O. selbst für diesen Fall auf.

d) Da der Schaden des Klägers auf einer Verletzung einer vertraglichen Nebenpflicht beruht, besteht eine gesetzliche Verschuldensvermutung nach § 280 BGB (vgl. auch OLG Düsseldorf, Urt. vom 02.08.1988 - 7 U 268/87 - NJW 1990, 2000). Sie zu entkräften, ist dem beklagten Land nicht gelungen. Vielmehr hat die Beweisaufnahme ergeben, dass die OFD jegliche Sicherungsmaßnahmen betreffend das Haus 2 unterlassen hatte. Das Land hat damit zumindest die im Verkehr erforderliche Sorgfalt nicht walten lassen und damit fahrlässig gehandelt, wobei es sich die Handlungen seiner Bediensteten zurechnen lassen muss. Dies gilt umso mehr, als der Zeuge P. glaubhaft darlegte, dass das Modell nur in den gänzlich ungesicherten Bereich verbracht wurde, um kurzfristigen Leerlauf am Bau, der den unterschiedlichen Arbeitszeiten der Hausmitarbeiter und der Handwerker geschuldet wäre, zu vermeiden. Notfalls hätte die OFD die Skulptur im Haus 3 belassen, dort von der einen zur anderen Stelle umsetzen und eine Verzögerung bei der Verlegung der Fußbodenplatten hinnehmen müssen.

e) Der Kläger muss sich weder ein Mitverschulden anrechnen noch eine die Haftung des beklagten Landes ausschließende schwerwiegende Eigenhaftung vorwerfen lassen. Weder war er bei Ausstellung des Modells verpflichtet, die Mitarbeiter des beklagten Landes über die allgemein bekannten Eigenschaften von Gips zu unterrichten, noch war er auf einen Hinweis des Zeugen O. auf Bauarbeiten in der OFD verpflichtet, Kontroll- und Schutzmaßnahmen zu ergreifen, denn dies hatte gerade das beklagte Land mit dem Leihvertrag übernommen.

f) Dem Schadensersatzanspruch des Klägers steht auch der vom beklagten Land behauptete Haftungsausschluss nicht entgegen. Den ihr hierfür obliegenden Beweis hat sie nicht führen können. Der Zeuge T. hat lediglich bestätigt, dass er den Kläger darauf hingewiesen habe, dass das Modell nicht versichert werden könne. Dass das beklagte Land stattdessen aber auch nicht aus eigener Kraft haften wolle, hat er nach eigenem Bekunden nicht erklärt. Das musste der Kläger auch nicht so verstehen.

g) Die Einrede der Verjährung des beklagten Landes greift nicht durch.

aa) Für den Fall, dass der Kläger Ansprüche wegen Veränderung und Verschlechterung des Kunstwerkes aus einem Leihverhältnis geltend machen will, findet die kurze Verjährungsfrist des $\S 606$ BGB Anwendung. Dies gilt nur dann nicht, wenn etwa wegen Zerstörung der Sache eine Unmöglichkeit der Rückgabe gegeben ist (BGH, MDR 1968, 401 = NJW 1968, 692; OLG, Köln Urt. vom 14.03.1995 - 22 U 202/94 - NJW 1997, 1157; OLG Düsseldorf, Urt. vom 30.07.1992 - 13 
U 268/91 - OLGR 1993, 19; Kollhosser in MünchKomm., 4. Aufl., § 606 Rn. 2; Reuter in Staudinger, Bearb. 2004, §606 Rn. 12).

Die Abgrenzung zwischen einer Verschlechterung und einer Zerstörung gestaltet sich insbesondere bei einem Kunstwerk schwierig. Eine Zerstörung oder Vernichtung des Kunstwerkes, welche der Anwendung des $\S 606$ BGB entgegen steht, soll nach der wohl herrschenden Meinung nicht vorliegen, wenn die Sache noch körperlich zurückgegeben werden kann (BGH NJW 1968, 694; BGH NJW 1981, 2406; OLG Oldenburg MDR 1982, 492; OLG Köln, Urt. vom 14.03.1995 - 22 U 202/94 - NJW 1997, 1157; OLG Düsseldorf, Urt. vom 02.08.1988 - 7 U 268/87 - NJW 1990, 2000; Reuter, a.a.O.; Kollhosser, a.a.O.). Darauf, ob es durch eine Reparatur wieder vollständig in seinen ursprünglichen Zustand versetzt werden kann, soll es dabei nicht ankommen (OLG Köln, a.a.O.), so dass auch der wirtschaftliche Totalschaden nur eine Verschlechterung im Sinne des $\S 606$ BGB darstellt (Reuter a.a.O.). So ging das OLG Köln in der vorzitierten Entscheidung von einer Verschlechterung bei der Einbeulung einer Bronzeskulptur aus, auch wenn bei deren Ausbeulen die Patina der Figur beschädigt würde. In dem Zerknicken, Einreißen und mit Klebstoffen Verunreinigen etc. von mehreren Gemälden liegt ebenfalls nur eine Beschädigung, noch keine Zerstörung (OLG Düsseldorf Urt. vom 02.08.1988 - 7 U 268/87 - NJW 1990, 2000). Die völlige Zerstörung eines Kraftfahrzeugs nahm das OLG Düsseldorf (Urt. vom 30.07.1992 - 13 U 268/91 - OLGR 1993, 19) an, wenn das Fahrzeug auch von einem Tüftler nicht ohne weiteres wieder aufgebaut werden kann, jedenfalls dies mindestens $150 \%$ des Wiederbeschaffungswertes erfordere, wobei dies ausdrücklich bei einem Kunstwerk anders zu beurteilen sein soll. Der BGH (NJW 1968, 694) hat bei einem wirtschaftlichen Totalschaden eines Fahrzeugs dagegen $\S 606$ BGB weiterhin für anwendbar gehalten. Das OLG Naumburg ging in seiner oben zitierten Entscheidung bei einem Knick in einer Metallskulptur auch von einer Beschädigung aus. Im Ergebnis dieser Rechtsprechung liegt eine Beschädigung solange vor, wie der Entleiher dem Verleiher auch nur das übrig gebliebene Material zurückgeben könne (Reuter in Staudinger, § 606 Rn. 12). Unter Anwendung dieser Grundsätze handelt es sich um eine Beschädigung des Kunstwerkes, da das beklagte Land das beschädigte Modell zurückgeben und der Kläger dieses durch Nachmodellierungen wieder vollenden kann, wenn es dann auch nicht das vollkommen gleiche Kunstwerk ist.

bb) Die Verjährung des Schadensersatzanspruches des Verleihers wegen der Verschlechterung der Leihsache beginnt grundsätzlich mit der Rückgabe derselben (OLG Köln, Urt. vom 14.03.1995 - 22 U 202/94 - NJW 1997, 1157; OLG Düsseldorf, Urt. vom 02.08.1988 - 7 U 268/87 - NJW 1990, 2000; Reuter a.a.O., § 606 Rn. 2; Kollhosser a.a.O., § 606 Rn. 3). Zurückgegeben hat das beklagte Land das Modell noch nicht. Es steht nach wie vor im Haus 3 der ehemaligen OFD. Statt der Rückgabe soll es aber auch genügen, wenn jedenfalls der Verleiher freiwillig auf die Übernahme der Sachherrschaft verzichtet (Reuter a.a.O., § 606 Rn. 2). So aber liegt der Fall hier nicht. Das beklagte Land, dass insoweit vortragsund beweispflichtig ist, hat lediglich vorgetragen, das der mit der Bauleitung der Umbaumaßnahmen von ihr beauftragte Architekt den Kläger über dessen Ehefrau aufgefordert habe, das beschä- digte Modell abzuholen, wobei der Wortlaut zwischen den Parteien streitig ist. Der Senat hegt bereits erhebliche Zweifel, ob der Architekt im Rahmen des Leihvertrages über eine entsprechende Vertretungsmacht verfügte, denn er war nach dem Vortrag des beklagten Landes mit der Bauleitung beauftragt, die in keinem unmittelbaren Zusammenhang mit dem Leihvertrag steht. Zudem hat der Kläger eine solche Befugnis bestritten.

Indes braucht der Senat dies nicht abschließend entscheiden. Grundsätzlich nämlich obliegt es dem Entleiher, die Leihsache am Sitz des Verleihers zurückzugeben (vgl. BGH NJW-RR 2002, 1027). Eine hiervon abweichende Vereinbarung ist weder ersichtlich noch vorgetragen. Sie folgt nicht schon aus der einseitigen Aufforderung des Entleihers, der Verleiher möge sie abholen. Sie begründet auch keinen Annahmeverzug des Klägers, der nur die Andienung der aus dem Vertrag geschuldeten Leistung bewirken kann. Daher vermag der Senat in dem Umstand, dass der Kläger einer solchen Aufforderung nicht nachgekommen ist, auch keinen auch nur konkludent erklärten Verzicht zu erkennen.

cc) Das beklagte Land hat dem Kläger das beschädigte Gipsmodell bis heute nicht in der vorgezeichneten Form zur Rücknahme angedient. Eine ausdrückliche Weigerung hierzu hat der Kläger ebenfalls nicht erklärt. Die Verjährung wurde damit noch nicht in Lauf gesetzt.

2. Schadensersatzansprüche aus $\S \S 823,826$ BGB braucht der Senat nicht zu prüfen, da diese über den Anspruch aus Verletzung des Leihvertrages nicht hinausgehen.

3. Neben diesem Anspruch kann der Kläger nicht mit Erfolg einen immateriellen Schadensersatzanspruch aus § 97 Urheberrechtsgesetz (UrhG) geltend machen.

Voraussetzung eines solchen Anspruches ist die Verletzung eines Urheberrechts oder sonst durch das Urheberrechtsgesetz geschützten Rechtes. Erfasst werden von § 97 UrhG daher nur die absoluten Urheberrechte und die ihnen gleichstehenden Rechte, die jedermann gegenüber bestehen (Möhring/Nicolini, a.a.O., § 97 Rn. 1; Dreyer/Kotthoff/Meckel: Urheberrecht, 2004, § 97 Rn. 1). Geschützt werden also die Rechte aus $\S \S 12$ bis 14, das Verwertungsrecht nach § 15 sowie Vorbereitungsrechte etc. nach §§ 17 ff. UrhG. Der Schutz besteht jedoch nur gegenüber der Beeinträchtigung dieser Rechte. Das heißt, hierher gehört etwa die Verletzung des Vervielfältigungsrechts des Urhebers durch die Vornahme von Vervielfältigungen durch einen nicht hierzu Befugten. Nicht unter den Schutzbereich des § 97 UrhG fallen relative Rechte, die einer bestimmten Person Pflichten auferlegen, z.B. aufgrund eines mit dieser Person geschlossenen Vertrages (Dreyer/Kotthoff/Meckel, a.a.O.). Hierher gehört es also nicht, wenn etwa durch die Verletzung vertraglicher Obhutspflichten Rechte verletzt oder Sachen beschädigt werden. Mangels entsprechender Normen im UrhG finden hierauf die Vorschriften des BGB, insbesondere etwa des $\S$ 280 BGB Anwendung (Loewenheim, a.a.O., § 62 Rn. 16). Das Interesse an der Erhaltung der Sachsubstanz ist durch das allgemeine bürgerliche Recht hinreichend geschützt. 